\title{
Neutrophil-lymphocyte ratio and response to plasmapheresis in Guillain-Barré syndrome: a prospective observational study
}

Noha Ali Hashim * (D), Wafaa Samir Mohamed and Engy Mohammed Emad

\begin{abstract}
Background: Guillain-Barré Syndrome (GBS) is one of the most severe neurological diseases that causes marked disability and even death.

Aim: The aim of this study is to investigate the role of the neutrophil-lymphocyte ratio (NLR) as a prognostic marker for GBS and response to treatment with plasmapheresis.

Methods: Seventy-five subjects (35 GBS patients and 40 healthy controls) were recruited. Complete general and neurological examinations were performed and Hughes disability scale score was evaluated for assessing functional motor deficits in GBS patients. In addition, NLR, erythrocyte sedimentation rate, and C-reactive protein level were calculated.

Results: NLR was significantly higher in GBS patients than in controls $(p<0.001)$ and was significantly higher in axonal form than other demyelinating and mixed subtypes $(p<0.02)$. Patients with a poor outcome had a significantly high NLR than patients with a good outcome $(p=0.006)$. NLR was also positively correlated with Hughes disability scale score $(p<0.001)$. The cut-off value for NLR to predict a good response of patients to plasmapheresis was $\leq 4.4$.

Interpretation: NLR may be a rapid, simple, inexpensive biomarker for predicting the severity of GBS, outcome of patients, and their response to plasmapheresis.
\end{abstract}

Keywords: Guillain-Barré syndrome, Neutrophil-lymphocyte ratio, Prognosis, Plasmapheresis

\section{Introduction}

Guillain-Barré Syndrome (GBS) is an acute, autoimmune, polyradiculoneuropathy [1]. The incidence of GBS is 0.81 to 1.89 per 100,000 populations [2] and it increases with age. Two peaks of incidence are noticed in late adolescence and young adulthood [3].

GBS presents clinically as acute progressive motor weakness, often with cranial nerve and sensory affection within 1 to 2 weeks after stimulation of the immune system and reaches the peak of clinical deficits in 2 to 4 weeks [4]. Respiratory failure occurs in $20 \%-30 \%$ of patients and is a life-threatening condition in GBS [5].

* Correspondence: n.hashim83@hotmail.com

Department of neurology, Faculty of Medicine, Zagazig University, El-Sharkia, Zagazig, Egypt
The most relevant theory for GBS is based on the molecular mimicry in which the body generates an immune response to incite factors mostly an infectious organism such as Campylobacter jejuni [6], Haemophilus influenza [7] or Cytomegalovirus that shares a determinate epitope with the host's affected tissues in GBS [8]. Both cellular and humoral immunity play a potential role in disease pathogenesis [9]. The immune response generates antibodies that cross react with gangliosides of the nerve membrane that cause nerve damage [10].

White blood cell count and its subtypes are wellknown markers of systemic inflammation. Neutrophils are main players in the innate immunity, and lymphocyte count is assumed to reflect the degree of responsiveness of the host's immune system [11]. 
Neutrophil-lymphocyte ratio (NLR) is calculated from the white blood cell count. This ratio has recently been known to be a strong predictor of systemic inflammation in many diseases such as sarcidosis, psoriasis, coronary heart diseases, and cancer [12]. A high NLR is suggested as a strong indicator of disease severity and poor prognosis in many neurological diseases such as acute ischemic stroke [13] and multiple sclerosis [14].

Estimating the progression of GBS and predicting the degree of severity and response to treatment are considered major challenges given that there are a very few reliable prognostic markers for GBS. The aim of this work it to evaluate the role of NLR as a prognostic marker of GBS in patients receiving plasmapheresis and highlight the relation between NLR and GBS severity.

\section{Subjects and methods}

\section{Patients}

This prospective study was conducted at Zagazig University Hospitals, Egypt. Thirty-five patients with GBS (20 women and 15 men) were enrolled. The clinical diagnosis of GBS was made according to Asbury and Cornblath's diagnostic criteria [15]. All 35 patients were subjected to detailed medical history and complete general and neurological examinations. All patients were treated with five sessions of plasmapheresis, one session every other day within 1 week from the onset of illness.

Exclusion criteria included patients less than 18 years old, those with concomitant inflammatory diseases, neoplasm, cardiac, hepatic, renal diseases, pregnancy, diabetes mellitus, and history of exposure to toxic substances or drugs that might affect the results of the nerve conduction study (e.g., chemotherapy). We also excluded patients who were admitted to the hospital after 5 days from the onset of symptoms.

Healthy 40 subjects matched for age and sex that visited the hospital for routine checkup were recruited as controls. The control subjects had no signs of peripheral neuropathy, and their laboratory investigations were not consistent with infection or chronic illness or inflammatory diseases.

\section{Clinical and electrophysiological assessments}

The clinical severity of GBS was assessed by using the GBS disability score (Hughes disability scale) [16]. Each patient was evaluated according to this scale at the time of admission ( 1 to 5 days after symptom onset) and 1 month later. We considered patients having a score of 0 or 2 within 1 month after admission to have a good outcome (good response to treatment) and those having a score of $\geq 3$ to have a poor outcome (no response to treatment).

Nerve conduction studies were conducted with an EMG machine (Nemus, Biomedica, Model number 00655, Galileo NT software version 3.71/00, Italy), within 1 week of onset. Patients were classified as having acute inflammatory demyelinating polyneuropathy (AIDP), acute motor-sensory axonal neuropathy (AMSAN), and acute motor axonal neuropathy (AMAN) by using the motor nerve conduction criteria [17].

\section{Blood sample collection}

The subjects underwent the following routine laboratory investigations: complete blood count, blood sugar, liver and kidney functions, and lipid profiles. A venous blood sample was collected under aseptic conditions from the subjects into ethylenediaminetetraacetic acid containing tubes at the time of admission (within 1 to 5 days from disease onset and before any treatment). A second sample was taken after three sessions of plasmapheresis within 7 days from the first venous sample. A complete blood count analysis was performed using flow cytometry. NLR was measured by dividing the neutrophil count by the lymphocyte count. C-reactive protein (CRP) level was determined by turbidometry. The erythrocyte sedimentation rate (ESR) was measured by the Westergren method. These parameters were determined and recorded.

The study was approved by the institutional ethics committee of the Faculty of Medicine, Zagazig University. Informed consent was obtained from all individual participants included in the study.

\section{Statistical analysis}

All statistical tests were performed using SPSS v. 20 (IBM SPSS Inc., Chicago, IL, USA) [18]. Qualitative data are presented as number $(N)$ and percentage (\%). Quantitative data are expressed as mean \pm standard deviation (SD). Chi-square test $\left(\chi^{2}\right)$ test was used for intergroup comparison. Spearman's correlation analysis was performed between the selected study parameters. Receiveroperating characteristic (ROC) curves were used to determine the cut-off values of NLRs. All tests were two sided and $p<0.05$ was considered statistically significant.

\section{Results}

\section{General characteristics of the studied population}

This study included 35 patients with GBS, 20 females (57.1\%) and 15 males (42.9\%), with a mean \pm SD age of $32.29 \pm 13.41$ years old and 40 normal healthy subjects as control group, 23 females (57.5\%) and 17 males (42.5\%), with a mean \pm SD age of $34.48 \pm 12.98$ years old. Demographic data and blood biochemistry values are summarized in Table 1 . There was no statistically significant difference between patients and controls regarding age, sex, and CRP level $(p>0.05)$.

ESR was statistically and significantly higher in GBS patients than in controls $(p=0.017)$.

The mean \pm SD value of the first observation of neutrophils (neutrophil 1) in GBS patients was $82.01 \pm$ 
Table 1 Demographic data, clinical characteristic and blood biochemistry values of the studied groups

\begin{tabular}{|c|c|c|c|}
\hline & Patients $(n=35)$ & Control $(n=40)$ & $p$ \\
\hline Age (years) & $32.29 \pm 13.41(18-65)$ & $34.48 \pm 12.98(18-70)$ & 0.47 \\
\hline \multicolumn{4}{|l|}{ Sex } \\
\hline Female & $20(57.1 \%)$ & $23(57.5 \%)$ & \multirow[t]{2}{*}{0.98} \\
\hline Male & $15(42.9 \%)$ & $17(42.5 \%)$ & \\
\hline CRP (mg/dl) & $6.46 \pm 4.9$ & $5.28 \pm 3.49$ & 0.31 \\
\hline $\mathrm{ESR}(\mathrm{mm} / \mathrm{h})$ & $28.43 \pm 18.47$ & $17.73 \pm 5.19$ & $0.017^{*}$ \\
\hline Neutrophil $1\left(10^{3} / \mathrm{mL}\right)$ & $82.01 \pm 13.64$ & $37.55 \pm 11.39$ & $<0.001^{*}$ \\
\hline Neutrophil $2\left(10^{3} / \mathrm{mL}\right)$ & $36.96 \pm 10.62$ & & 0.82 \\
\hline Lymphocyte $1\left(10^{3} / \mathrm{mL}\right)$ & $22.59 \pm 7.5$ & $15.31 \pm 5.34$ & $<0.001^{*}$ \\
\hline Lymphocytes $2\left(10^{3} / \mathrm{mL}\right)$ & $16.83 \pm 3.83$ & & 0.17 \\
\hline NLR 1 & $3.95 \pm 1.34$ & $2.58 \pm 0.85$ & $<0.001^{*}$ \\
\hline NLR 2 & $2.27 \pm 0.76$ & & 0.10 \\
\hline Hughes disability scale score 1 & $3.28 \pm 1.29$ & - & \\
\hline Hughes disability scale score 2 & $1.92 \pm 1.77$ & - & \\
\hline \multicolumn{4}{|l|}{ History of infection: } \\
\hline Yes & $20(57.1 \%)$ & - & \\
\hline No & 15 (42.9\%) & - & \\
\hline
\end{tabular}

Data were expressed as number $(N)$ and percentage (\%),

NLR1 neutrophil-lymphocyte ratio before plasmapheresis, NLR2 neutrophil-lymphocyte ratio after plasmapheresis, ESR erythrocyte sedimentation rate, CRP C-reactive protein

* Statistically significant at $p<0.05$

$13.64 \times 10^{3} / \mathrm{mL}$ and it was statistically and significantly higher in patients than in controls $(37.55 \pm 11.39) 10^{3} /$ $\mathrm{mL} ; p<0.001$. The mean \pm SD of the second observation of neutrophils in patients (neutrophil 2) after three sessions of plasmapheresis was markedly decreased $\left(36.96 \pm 10.62 \times 10^{3} / \mathrm{mL}\right)$ with no statistically significant difference between the groups ( $p=0.82$ ).

In addition, the first observation of lymphocytes in patients before treatment (lymphocytes 1, 22.59 $\pm 7.5 \times$ $10^{3} / \mathrm{mL}$ was statistically and significantly higher in patients than controls $\left(15.31 \pm 5.34 \times 10^{3} / \mathrm{mL}, p<0.001\right)$; however, after three sessions of plasmapheresis, the second observation of lymphocytes in patients (lymphocytes $2,16.83 \pm 3.83 \times 10^{3} / \mathrm{mL}$ ) showed no statistically significant difference between the groups $(p=0.17)$.

For NLR before plasmapheresis (NLR1), its mean \pm SD value in patients $(3.95 \pm 1.34)$ had a statistically significant high difference with controls $(2.58 \pm 0.85, p<0.001)$. After three sessions of plasmapheresis, the second NLR value (NLR 2$)$ was markedly decreased $(2.27 \pm 0.76)$ with no statistically significant difference between the groups $(p=0.10)$. The mean \pm SD Hughes disability score of patients at admission (Hughes score 1) was $3.28 \pm 1.29$ and the score at follow-up evaluation after 1 month was $1.92 \pm 1.77$. Twenty patients (57.1\%) had a history of infection before the onset of GBS. Eight patients (22.9\%) had diarrhea and 12 (34.2\%) patients experienced an upper respiratory tract infection within the last 2 weeks before onset of neuropathy.

\section{Clinical characteristic of patients and NLR before and after plasmapheresis}

All patients are classified according to their electrophysiological finding to 18 patients (51.4\%) with AIDP, 7 (20\%) with AMAN, and 10 (28.6\%) with AMSAN. The NLR value was statistically significantly higher in AMSAN subtype of GBS than in other types of GBS before plasmapheresis $(p<0.02)$; the value decreased significantly after plasmapheresis but was still higher in AMSAN subtype but without any statistical significance $(p=0.18)$.

Patients having a history of infection before illness had no significant difference from those without infection regarding NLR, either before or after plasmapheresis ( $p=$ 0.12 and $p=0.80$, respectively). However, both groups showed a significant reduction in NLR after three sessions of plasmapheresis $(p=0.001, p<0.001$; Table 2$)$.

\section{NLR and response to plasmapheresis treatment}

All 35 patients received plasmapheresis within 1 week from the onset of illness as a basic therapy. Only 26 patients $(74.28 \%)$ showed a good response. Nine patients (25.71\%) continued their treatment with intravenous immunoglobulin (IVIG) because of deterioration of the condition in spite of five sessions of plasmapheresis, and only one patient died from respiratory failure. Between the 26 patients and the 9 patients, there was no statistically significant difference regarding age, sex, Hughes disability scale score, CRP level, ESR, and NLR2. Only 
Table 2 The association between clinical characteristic and pretreatment/post-treatment NLR values

\begin{tabular}{lllll}
\hline & $N(\%)$ & NLR 1 & NLR 2 & $p$ \\
\hline Type & & & & \\
AIDP & $18(51.4 \%)$ & $3.47 \pm 1.04$ & $1.98 \pm 0.45$ & $<0.001^{*}$ \\
AMAN & $7(20 \%)$ & $4.04 \pm 1.85$ & $2.17 \pm 0.61$ & $<0.02^{*}$ \\
AMSAN & $10(25.7 \%)$ & $4.94 \pm 1.08$ & $2.51 \pm 1.06$ & $<0.001^{*}$ \\
& & $P<0.02^{*}$ & $P<0.18$ & \\
History of infection & & & \\
No & $15(42.9 \%)$ & $3.67 \pm 1.22$ & $2.21 \pm 0.63$ & $<0.001^{*}$ \\
Yes & $20(57.1 \%)$ & $4.33 \pm 1.45$ & $2.34 \pm 0.92$ & $0.001^{*}$ \\
& & $P 0.12$ & $P 0.80$ & \\
\hline
\end{tabular}

AIDP acute inflammatory demyelinating polyneuropathy, AMSAN acute motorsensory axonal neuropathy, AMAN acute motor axonal neuropathy, NLR1: neutrophil-lymphocyte ratio before plasmapheresis, NLR2 neutrophillymphocyte ratio after plasmapheresis

*Statistically significant at $p<0.05$

the early NLR was significantly higher in the 9 patients than in 26 patients (Table 3).

Correlation between Hughes disability scale score and clinical laboratory findings of our GBS patients showed that only NLR1 positively correlated with a bad outcome $(p<0.001$, Table 4$)$.

The cut-off value for NLR for its ability to predict response to plasmapheresis was $\leq 4.4$ with $81.5 \%$ sensitivity and $87.5 \%$ specificity. The ROC area under the curve (AUC) was 0.85 [confidence interval (CI) $1.69-0.95, p<0.001$ ]. A CRP value of $\leq 1$ had $18.5 \%$ sensitivity and $90 \%$ specificity for predicting response to plasmapheresis (CI $0.33-0.67$, AUC $0.50, p=1.1$ ). An ESR value of $>16$ had $77.8 \%$ sensitivity and $50 \%$ specificity for predicting response to plasmapheresis (CI 0.39-0.73, AUC 0.56, $p=0.57$; Fig. 1).

\section{Discussion}

GBS is considered as one of the most common and devastating paralytic neuropathies with a fatality rate of 5\%-
Table 4 Correlation between clinical parameter and Hughes disability scale

\begin{tabular}{llllll}
\hline & \multicolumn{2}{l}{ Hughes scale scores 1 } & & \multicolumn{2}{l}{ Hughes scale scores 2 } \\
\cline { 2 - 3 } & $r$ & & & $r$ & $p$ \\
\hline NLR1 & 0.54 & $<0.001^{*}$ & & 0.55 & $<0.001^{*}$ \\
ESR $(\mathrm{mm} / \mathrm{h})$ & -0.036 & 0.42 & & 0.106 & 0.27 \\
CRP $(\mathrm{mg} / \mathrm{dl})$ & -0.036 & 0.14 & & 0.079 & 0.33 \\
NLR2 & - & - & & 0.32 & 0.06 \\
\hline
\end{tabular}

NLR1 neutrophil-lymphocyte ratio before plasmapheresis, NLR2 neutrophillymphocyte ratio after plasmapheresis, ESR erythrocyte sedimentation rate, CRP C-reactive protein

*Statistically significant at $p<0.05$

$10 \%$. Because of a wide variety of clinical manifestations of GBS, predicting clinical severity and outcomes is necessary to help clinicians modify supportive care and treatment to patients [2].

This study is aimed to evaluate the role of NLR as a prognostic marker in GBS and highlight the relation among NLR and GBS severity and response to plasmapheresis.

NLR is a combination of two markers. It is suitable than other leukocyte parameters because of its stability compared with total leukocyte count, and neutrophil and lymphocyte counts which could change by various physiological and pathological factors [19]. In addition, elevated NLR could be observed in inflammatory diseases without significant leukocytosis [20]. NLR is easily calculated and inexpensive than other inflammatory markers [21].

In our study, we found that the initial serum NLR values were significantly higher in GBS patients than in controls $(p<0.001)$. These findings are consistent with the results of Geyik et al. [22] and Ozler and Gunak, [23]. To highlight the relation between GBS and NLR, Hou et al. [24] reported that GBS is an acute inflammatory disease caused by $\mathrm{T}$ cell-mediated autoimmunity to

Table 3 Comparison between patients showing good response to treatment and those not responding to plasmapheresis

\begin{tabular}{|c|c|c|c|}
\hline & Patients with good outcome (26) & Patients with bad outcome (9) & $p$ \\
\hline Age (years) & $29.85 \pm 11.49$ & $39.3 \pm 16.61$ & 0.07 \\
\hline \multicolumn{4}{|l|}{ Sex } \\
\hline Male & $12(46.2 \%)$ & $3(33.3 \%)$ & \multirow[t]{2}{*}{0.5} \\
\hline Female & $14(53.8 \%)$ & $6(66.7 \%)$ & \\
\hline Hughes disability scale scores 1 & $3.3 \pm 1.15$ & $3 \pm 1.6$ & 0.5 \\
\hline Hughes disability scale scores 2 & $1.54 \pm 1.36$ & $1.3 \pm 1.8$ & 0.7 \\
\hline NLR 1 & $3.60 \pm 1.18$ & $5.11 \pm 1.78$ & $0.006^{*}$ \\
\hline NLR 2 & $2.10 \pm 0.66$ & $2.83 \pm 0.84$ & 0.05 \\
\hline CRP $(\mathrm{mg} / \mathrm{dl})$ & $6.5 \pm 5.42$ & $6.3 \pm 2.92$ & 0.92 \\
\hline $\operatorname{ESR}(\mathrm{mm} / \mathrm{h})$ & $27.1 \pm 19.07$ & $32.3 \pm 17.04$ & 0.47 \\
\hline
\end{tabular}

NLR1 neutrophil-lymphocyte ratio before plasmapheresis, NLR2 neutrophil-lymphocyte ratio after plasmapheresis, ESR erythrocyte sedimentation rate, CRP C-reactive protein

*Statistically significant at $p<0.05$ 


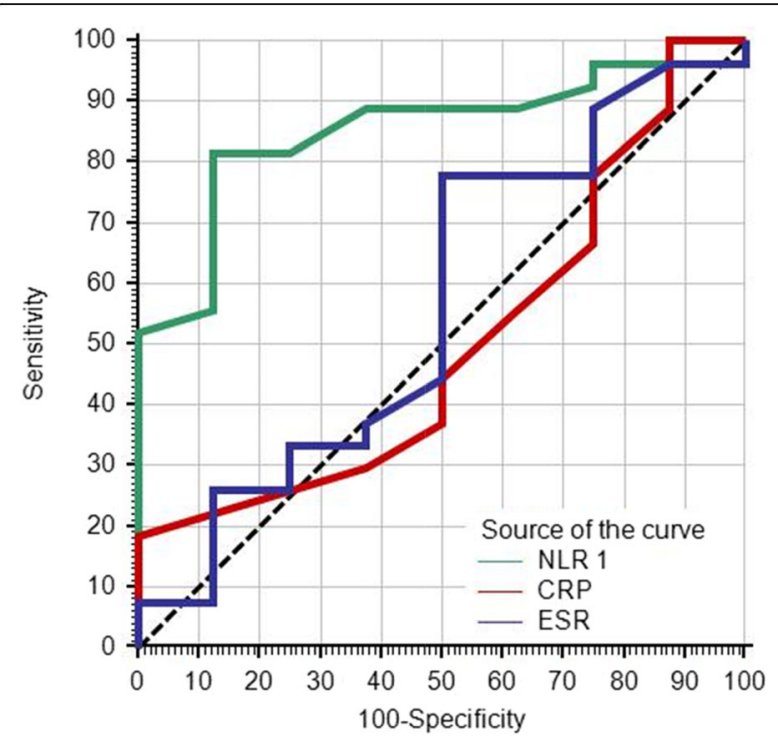

Fig. 1 ROC curve analysis of NLR, ESR, and CRP in the prediction of response to plasmapheresis. NLR neutrophil-lymphocyte ratio, ESR erythrocyte sedimentation rate, CRP C-reactive protein

myelin proteins and autoantibody-mediated humoral immunity to myelin glycolipids. They added that NLR was simple and cost-effective biomarkers that pointed to the inflammatory process of GBS. Demirci et al. [25] proposed that NLR which represents the balance between neutrophils and lymphocytes is an informative noninvasive marker to detect systemic inflammatory conditions. Nishida et al. [26] reported that neutrophils which are one of the most important mediators of the innate immunity consecrate phagocytes that augment the acute inflammatory reaction and form the first line of defense mechanism against invading pathogens. Neutrophils are important leukocytes that trigger inflammation and cause marked tissue injury.

In our study, when we compared the three electrophysiological variants of GBS, we found that the initial NLR values were higher in the AMSAN subtype $(p<$ 0.02 ) than those in other subtypes. In the axonal form, the first immune response is directed toward the node of Ranvier causing functional axonal impairment by alternating ions and water homeostasis and sodium channel dysfunction [23]. Axon damage produces hundreds of times the normal amount of two chemokines CXCL1 and CXCL2 which attach to the surface of neutrophils and draw immune cells into injured tissues. The neutrophils engulf cellular debris tidying up the area for nerve repair [27].

To clarify the role of NLR in the prediction of GBS prognosis, we found that patients with a poor outcome had a significantly higher initial NLR1 value than patients with a good outcome $(p=0.007)$, and after plasmapheresis, NLR2 was still significantly high in patients with a poor outcome $(p=0.01)$. In addition, our results revealed a significant positive correlation between the initial NLR values and the mean Hughes disability scale scores of both types of patients at presentation $(p<0.001)$, and at the end of follow-up $(p<0.001)$. Our findings agreed with those of Geyik et al. [22] who concluded that NLR values positively and significantly correlated with disease severity, and the authors added that this ratio might be a promising marker in GBS. Sahin et al. [20] reported that a high NLR was significantly related to initial disability in GBS patients. The NLR value positively correlated with the grade of facial paralysis [23]. In GBS, neutrophils secrete pro-inflammatory enzymes and reactive oxygen species that could exacerbate inflammation and cause massive tissue injury [28]. In addition, these cells could produce type 1 interferon and tumor necrosis factor that induce B cell proliferation. B cells produce several inflammatory cytokines, autoantibodies, and oxidative species that can damage the myelin sheath and cause nerve dysfunction [29]. In severe cases, the motor axon might undergo Wallerian degeneration leading to delay in recovery [2]. Hiraga et al, [30] reported that antigens are located on the myelin sheath in the demyelinating variant of GBS. Autoantibodies activate the complement leading to the formation of membrane attack complex on the outer surface of Schwann cells, initiation of vesicular myelin degeneration and invasion of myelin by macrophages. These macrophages are recruited to strip off the myelin sheath and with severe inflammation, axonal loss could occur.

To evaluate the therapeutic role of plasmapheresis in GBS and its relation to NLR, we found that the cut-off value for the NLR for predicting response to plasmapheresis in GBS was $\leq 4.4$ with $81.5 \%$ sensitivity and $87.5 \%$ specificity $(p<0.001)$. Plasma exchange could remove neurotoxic antibodies, complement factors, cytokines, immune complex, and other inflammatory mediators [31]. To our knowledge, no study has yet established the relation between NLR and response to plasmapheresis in GBS.

In the present study, ESR was significantly higher in GBS patients than in controls $(p=0.017)$. In addition, CRP was higher in GBS patients than controls but without any statistically significance $(p=0.31)$. Both ESR and CRP had no correlation with the disease severity or response to plasmapheresis. CRP and ESR are nonspecific markers for any inflammatory disease or tissue damage $[32,33]$.

\section{Summary}

Our results show that NLR could be considered a new simple, rapid, and inexpensive marker of inflammation in GBS patients. The baseline peripheral blood NLR could be used as a practical and reliable 
prognostic marker for GBS patients and their response to plasmapheresis. By using NLR ratio in patients with GBS, more appropriate clinical management can be implemented, especially for those with a high NLR who will require more aggressive treatment.

\section{Abbreviations}

AIDP: Acute inflammatory demyelinating polyneuropathy; AMAN: Acute motor axonal neuropathy; AMSAN: Acute motor-sensory axonal neuropathy; CRP: C-reactive protein; ESR: Erythrocyte sedimentation rate; GBS: GuillainBarré syndrome; IVIG: Intravenous immune globulin; NLR: Neutrophillymphocyte ratio

\section{Acknowledgements}

The authors would like to appreciate all participants and their families as well as the hospital staff who contributed to the study.

\section{Authors' contributions}

$\mathrm{NAH}$, WSM, and EME collected the patients, gathered clinical data, wrote the manuscript, designed the study, and did the statistical analysis. All authors were involved in drafting the article or revising it critically for important intellectual content, and all authors read and approved the final version to be published.

\section{Funding}

This study was not supported by any source of finding.

\section{Availability of data and materials}

Data and materials supporting the results of this article are included within the article (and its additional file(s)).

\section{Ethics approval and consent to participate}

The study was approved from the Institutional Ethics Committee of the Faculty of Medicine, Zagazig University (ZU-IRB \#5013/18-12-2018). Written informed consent was obtained from all study participants after explaining the details and benefits as well as risks to them. Surrogate consent from the patient's legal guardian or designated health proxy was permitted in cases where the patient did not have decision-making capacity.

\section{Consent for publication}

Consent has been obtained from the participants involved in the study to report their individual patient data.

\section{Competing interests}

The authors declared that they have no conflicts of interest with respect to the authorship and/or publication of this article.

\section{Received: 2 December 2019 Accepted: 13 January 2020}

Published online: 30 January 2020

\section{References}

1. Willison HJ, Jacobs BC, van Doorn PA. Guillain-Barré syndrome. Lancet. 2016; 388:717-27.

2. Van den Berg B, Walgaard C, Drenthen J, et al. Guillain-Barr'e syndrome: pathogenesis, diagnosis, treatment and prognosis. Nat Rev Neurol. 2014; 10(8):469-82.

3. Newswanger DL, Warren CR. Guillain-Barré syndrome. Am Fam Physician. 2004:69(10):2405-10.

4. Fokke C, van den Berg B, Drenthen J, et al. Diagnosis of Guillain-Barré syndrome and validation of Brighton criteria. Brain. 2014;137:33-43.

5. Dhar R, Stitt L, Hahn AF. The morbidity and outcome of patients with Guillain-Barre syndrome admitted to the intensive care unit. J Neurol Sci. 2008:264(1-2):121-8

6. Yuki N, Susuki K, Koga M. Carbohydrate mimicry between human ganglioside GM1 and Campylobacter jejuni lipooligosaccharide causes Guillain-Barré syndrome. Proc Natl Acad Sci. 2004;101:11404-9.

7. Ju YY, Womersley H, Pritchard J, et al. Haemophilus influenzae as a possible cause of Guillain-Barré syndrome. J Neuroimmunol. 2004;149(1-2):160-6.
8. Yuki N, Yamada M, Koga M, et al. Animal model of axonal Guillain-Barré syndrome induced by sensitization with GM1 ganglioside. Ann Neurol. 2001;49:712-20

9. Dieleman J, Romio S, Johansen K, et al. Guillain-Barre syndrome and adjuvanted pandemic influenza A (H1N1) 2009 vaccine: multinational casecontrol study in Europe. BMJ. 2011;6:343-7.

10. Ozdemir $\mathrm{H}$. Analysis of the albumin level, neutophil/ lymphocyte ratio and platelet/ lymphocyte ratio in Guillain-Barré syndrome. Arq Neuropsiquiatr. 2016:4(9):718-22

11. Segal A. How neutrophils kill microbes. Ann Rev Immunol. 2005;23:197-223

12. Salciciolli JD, Marshall DC, Pimentel MAF, et al. The association between the neutrophil-to-lymphocyte ratio and mortality in critical illness: an observational cohort study. Crit Care. 2015:19:13-21.

13. Tokgoz S, Kayrak M, Akpinar Z, et al. Neutrophil lymphocyte ratio as a predictor of stroke. J Stroke Cerebrovasc Dis. 2013:22:1169-74.

14. Bisgaard AK, Pihl-Jensen G, Frederiksen JL. The neutrophil-to-lymphocyte ratio as disease actvity marker in multiple sclerosis and optic neuritis. Mult Scler Relat Disord. 2017;18:213-7.

15. Asbury K, Cornblath D. Assesment of current diagnostic criteria for Guillain Barre syndrome. Ann Neurol. 1990;27:21-4.

16. Walgaard $C$, Lingsma $H$, van Doorn $P$, Jacobs $B$. Early recognition of poor prognosis in Guillian Barre syndrome. Neurology. 2011;76:968-75.

17. Hadden RD, Cornblath DR, Hughes RA, et al. Electrophysiological classification of Guillain-Barré syndrome: clinical associations and outcome. Plasma exchange/Sandoglobulin Guillain-Barré syndrome trial group. Ann Neurol. 1998:44(5):780-8.

18. Levesque R. SPSS programming and data management: a guide for SPSS and SAS users. 4th ed. Chicago: SPSS Inc; 2007. ISBN 1-56827-390-8

19. Nunez J, Nunez E, Bodi $V$, et al. Usefulness of the neutrophil to lymphocyte ratio in predicting long term mortality in ST segment elevation in myocardial infarction. Am J Cardiol. 2008:101(6):747-52.

20. Sahin S, Cinar N, Karsidag S. Are cerebrospinal fluid protein levels and plasma neutrophil/ lymphocyte ratio associated with prognosis of GuillainBarré syndrome. Neurol Int. 2017;9:7032-6.

21. Turkmen $\mathrm{K}$, Tonbul $\mathrm{HZ}$, Toker $\mathrm{A}$, et al. The relationship between oxidative stress, inflammation, and atherosclerosis in renal transplant and end-stage renal disease patients. Ren Fail. 2012;34:1229-37.

22. Geyik S, Bozkurt H, Neyal M, et al. The clinical significance of the neutrophilto-lymphocyte ratio in patients with Guillain-Barré syndrome independent of infection. Med Sci Discov. 2016;3(8):305-11.

23. Ozler G, Gunak G. Neutrophil-lymphocyte ratio: a new predictive and prognostic factor in patients with Bell palsy. J Craniofac Surg. 2014;25:944-5

24. Hou H, Miao J, Dan FX, et al. Changes in lymphocyte subsets in patients with Guillain-Barré syndrome treated with immunoglobulin. BMC Neurol. 2014;14:202-9.

25. Demirci S, Kutluhan S, Rifat H, Yurekli V. The clinical significance of neutrophil-to-lymphocyte ratio in multiple sclerosis. Int J Neurosci. 2015; 126(8):700-6.

26. Nishida Y, Hosomi S, Yamagami H, et al. Neutrophil-to-lymphocyte ratio for predicting loss of response to infliximab in ulcerative colitis. PLoS ONE. 2017;12(1):e0169845.

27. Lindborg JA, Mack M, Zigmond RE. Neutrophils are critical for myelin removal in a peripheral nerve injury model of Wallerian degeneration. Neurosci. 2017;37(43):10258-77.

28. Steinbach $K$, Piedavent M, Bauer S, et al. Neutrophils amplify autoimmune central nervous system infiltrates by maturing local APCs. J Immunol. 2013; 191(9):4531-9.

29. Dimachkie M, Barohn R. Guillain-Barre' syndrome and variants. Neurol Clin. 2013:31(2):491-510.

30. Hiraga $A$, Mori $M$, Ogawara $K$, et al. Differences in patterns of progression in demyelinating and axonal Gullain-Barre syndrome. Neurology. 2003:61(4):471-4.

31. Hughes R. Plasma exchange versus intravenous immunoglobulin for Guillain-Barre'syndrome. Ther Apher. 1997;1:129-32.

32. Morley JJ\& Kushner I. Serum C-reactive protein levels in disease. Annals NY Acad Sci. 1982;389:406-18.

33. Susana E, Booker TB. Erythrocyte sedimentation rate from folklore to facts. Am J Med. 1985;78(6):1001-9.

\section{Publisher's Note}

Springer Nature remains neutral with regard to jurisdictional claims in published maps and institutional affiliations. 